\title{
Alejandro ÁLVAREZ-NOBELL*
}

Desarrollo cultural en las organizaciones. Un modelo de estudio basado en la Grounded Theory ${ }^{l}$

Cultural development in organizations. A model study based on the Grounded Theory

\section{María ROMERO-CALMACHE}

\section{Manuel SÁNCHEZ-SANVICENTE Paz ARAGÜÉS-DUFOL}

\section{Resumen}

La cultura organizacional se configura a partir de la interrelación de los procesos de apropiación de la filosofía, la pertenencia, la adaptación, la satisfacción y el liderazgo compartidos por un grupo. Este conjunto de categorías puede ser reconocido mediante el uso de una matriz que incluye en su estructura subcategorías o conceptos y un conjunto de propiedades observables en el público interno. El presente artículo tiene por objetivo describir un modelo de estudio construido a partir de la Grounded Theory o Teoría Fundamentada que nos permita comprender el desarrollo cultural de las organizaciones. El estudio de caso se realizó en una compañía líder en Europa del sector de la distribución.

\section{Palabras clave}

Comunicación; Cultura Organizacional; Clima Organizacional; Teoría Fundamentada

\section{Sumario}

1. Introducción. 2. La cultura organizacional: fundamentos y bases teóricas. 3. Metodología. 4. Resultados. 4.1 Matriz de desarrollo cultural organizacional. 4.1 Filosofía. 4.2 Adaptación. 4.3 Pertenencia. 4.4 Liderazgo. 4.5 Satisfacción. 5. Conclusiones. 6. Agradecimientos. 7. Referencias bibliográficas.

\begin{abstract}
The organizational culture is set from the interplay of the processes of appropriation of philosophy, membership, adaptation, satisfaction and leadership shared by a group. This set of categories can be recognized by using a matrix that includes in its structure or sub-concepts and a set of observable properties in the workforce. This article aims to describe a study model built from the Grounded Theory that allows us to understand the cultural development of organizations. The case study was conducted in a European leader in the distribution sector.
\end{abstract}

\section{Keywords}

Communication; organizational culture; working atmosphere; grounded theory

\footnotetext{
Summary

1. Introduction. 2. Organizational culture: rationale and theoretical basis. 3. Methodology. 4. Results. 4.1 Matrix organizational cultural development. 4.1 Philosophy. 4.2 Adaptation. 4.3 Membership. 4.4 Leadership. 4.5 Satisfaction. 5. Conclusions. 6. Acknowledgements. 7. References.
}

1 Estudio realizado por Felicicom Lab (Laboratorio de Comunicación Organizacional, Persuasión y Felicidad). Grado de Publicidad y Relaciones Públicas de la Facultad de Comunicación de la Universidad San Jorge (Zaragoza, España). http://felicicomlab.usj.es/ 


\section{Introducción}

El presente artículo tiene por objetivo describir un modelo de estudio que nos permita comprender el desarrollo cultural de las organizaciones partiendo del análisis de datos obtenidos de la realidad e identificando allí proceso sociales básicos que son de nuestro interés (Strauss, 1990). Su elaboración se enmarca en la Grounded Theory o Teoría Fundamentada, formulada por los sociólogos Barney G. Glaser y Anselm L. Strauss de la Universidad de Columbia en 1976 y de creciente aplicación en el campo científico de la comunicación y el marketing (Soler Pujals y Fernández Gómez, 2010). A través de esta metodología de análisis unida al trabajo de campo realizada en una empresa líder del sector de la distribución en Europa; se empleó un conjunto de métodos sistemáticamente aplicados para generar una teoría inductiva sobre el desarrollo cultural de las organizaciones (Glaser, 1992).

Inicialmente realizaremos una contextualización teórica del concepto de cultura en la organización; luego la descripción detallada -por la relevancia que tiene a los fines de comprender los resultados- del diseño metodológico y el caso empleado; para finalizar con el modelo de estudio sobre el desarrollo cultural en las organizaciones.

\section{La cultura organizacional: fundamentos y bases teóricas}

El término cultura proviene del verbo latino colere que designaba la acción de labrar la tierra. Durante siglos se ha mantenido dicha acepción hasta que en el XVII comienza a usarse para referirse al 'cultivo del conocimiento'. No es hasta el siglo XX cuando se le otorga el significado coloquial que ahora se conoce.

En términos generales, podemos definir la cultura como el sistema de comportamiento transmitido socialmente, que sirve para relacionar las comunidades con el medio en el que se desenvuelven. Numerosas son las investigaciones que abarcan la cultura como objeto de estudio puesto que se ha demostrado que, históricamente, este constructo ha supuesto un elemento estructural de todas las sociedades, sin distinción de procedencia ni de tiempo. La cultura supone una vía de adaptación del hombre a sus circunstancias, pero también implica una serie de comportamientos que lo identifican y lo diferencian.

El estudio de la cultura, como estructura y sistema social, tiene sus raíces en las investigaciones de las ciencias antropológicas. Abravanel, Allaire y Firsirotu (1992) realizaron una distinción fundamental entre dos corrientes de 
pensamiento que explicaremos de forma somera para contextualizar la investigación realizada: la que considera a la cultura como parte integrante del sistema de ideas y la que la ve como un sistema socio-cultural.

La escuela que entiende la cultura como un sistema sociocultural, se divide en cuatro corrientes: funcionalista, funcionalista-estructuralista, ecológico-adaptacionista e histórico-difucionista. Por otro lado, la cultura vista como sistema de ideas, está representada por cuatro perspectivas: la cognoscitiva, la estructuralista, la de equivalencia mutua y la simbólica. La suma de todos estos estudios da como resultado una visión amplia y poliédrica de un concepto especialmente complicado de definir por cuanto abarca elementos difícilmente calificables. La cultura llega a ser un contexto dinámico cargado de símbolos, un conjunto de cogniciones funcionales o una estructura mental profunda y subconsciente.

González Romo desarrolla una lista de características que posee el concepto de cultura y que nos sirven para terminar de dar forma a la idea expuesta en líneas anteriores:

- "La cultura surge por los intereses de un grupo social de adaptarse a un medio ambiente para poder sobrevivir.

- La cultura está formada por conocimientos que surgen de ese proceso de adaptación.

- Dichos conocimientos son transmitidos de generación en generación y cultivados históricamente.

- La cultura se basa en las creencias, costumbres, actitudes y hábitos de un grupo social determinado.

- El grupo social en el que se manifiesta la cultura posee una particular forma de comunicación basada en un lenguaje específico y en unos símbolos que todo el grupo debe interpretar" (2002: 90-91).

Figura $N^{0}$ 1: Modelos teóricos del estudio de la cultura

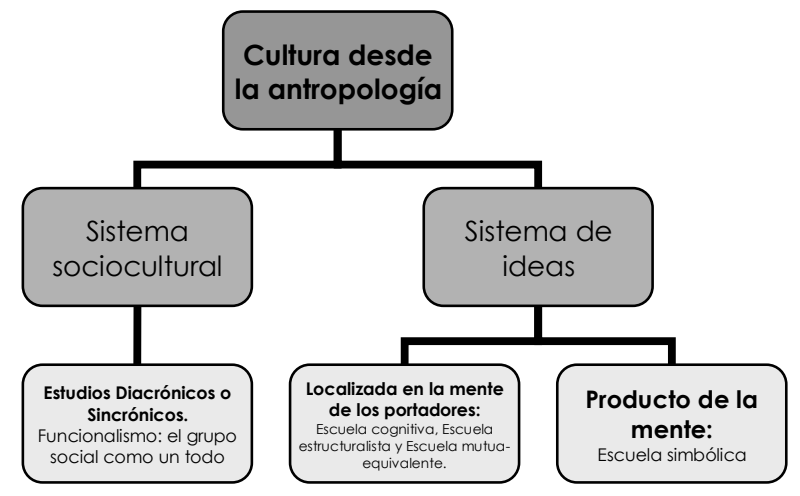

Fuente: Sánchez, 2000: 324-325. 
1 tal como las denominan Stefanova y Lucas, subculturas (2006).

2 La sociología de la empresa, la psicología, la antropología o las recientes teorías del Management.
En otro orden de cosas, los sociólogos aplican entrevistas sistemáticas, cuestionarios y otros métodos cuantitativos de recolección de datos que permiten obtener tipologías claras de atributos culturales. Estas técnicas y herramientas pueden aplicarse eficazmente para analizar la cultura de diferentes instituciones. Las empresas nacen de la organización y el trabajo como notas indispensables que constituyen un todo único. Son entes sociales ${ }^{1}$ en los que el hombre, además de aportar su fuerza de trabajo, se desarrolla y relaciona por lo que es fácilmente comprensible que en cada entorno se genere un sistema de ideas propio, una expresión particular del pensamiento empresarial y una forma peculiar de trabajar. En esta línea, los estudios se han desarrollado desde diferentes perspectivas y disciplinas ${ }^{2}$ y en distintos momentos históricos comenzando a principios del siglo XVIII.

Si bien es difícil establecer una definición certera y universal de la cultura organizacional, sí es posible repasar varias de las ideas que más aceptación han tenido en entornos académicos y empresariales y que son un claro reflejo de la importancia que puede adquirir la cultura organizacional en la práctica. Rodríguez de San Miguel define a la cultura organizacional como "el conjunto de valores y creencias comúnmente aceptados, consciente o inconsciente, por los miembros de una organización" (2003: 53). Aunque es probable que la definición más influyente sea la de Edgar Schein, por ser el autor que mayor difusión le ha dado al concepto: "Llamaré cultura a un modelo de presunciones básicas-inventadas, descubiertas o desarrolladas por un grupo dado, al ir aprendiendo a enfrentarse con sus problemas de adaptación externa e integración interna-; que hayan ejercido la suficiente influencia como para ser consideradas válidas, y en consecuencia, ser enseñadas a los nuevos miembros, como el modo correcto de percibir, pensar y sentir esos problemas" (Schein, 1998: 25).

Por su parte, Gagliardi añade que "la cultura distingue a una organización de otra y orienta sus decisiones", lo que quiere decir que le confiere su identidad (aquello que le hace ser lo que es) y define su propio estilo de hacer frente a los problemas derivados de su funcionamiento interno y de su adaptación externa (1984: 21).

Se puede visualizar que las definiciones anteriormente mencionadas tienen varios elementos comunes, tales como: creencias, valores, normas, que son compartidos por un grupo y que condicionan su comportamiento. Por lo tanto, la cultura organizacional debe ser entendida a los fines de esta 
3 Entiéndase como elementos interactivos a la interrelación entre las manifestaciones, valores, creencias y supuestos básicos. investigación como el conjunto de valores, creencias y supuestos básicos compartidos por un grupo, sobre los cuales se construye el decidir organizacional, que identifica a una organización y la diferencia de otra, haciendo que sus miembros se sientan parte de ella.

Antes de concluir el proceso de definición de la cultura organizacional, es necesario mencionar varias cuestiones fundamentales que encierra este concepto:

- La definición de la cultura da cuenta de un conjunto de elementos interactivos ${ }^{3}$ fundamentales, generados $y$ compartidos por los miembros de un grupo $u$ organización.

- La cultura se genera por la interacción al tratar de alcanzar los objetivos grupales. Las experiencias generadas y luego compartidas, construyen la cultura organizacional.

- Al ser "compartida" la cultura, genera identidad y cohesión interna en la organización.

En definitiva, el concepto se centra en la manera en la que los grupos interpretan, construyen y negocian la realidad a través de la creación de una cultura organizacional. La cultura, por tanto, constituye el contexto para la interpretación de un sistema de significados dentro del cual tiene lugar la interacción social.

\section{Metodología}

Para poder describir un modelo de estudio que nos permitiese comprender el proceso de implantación y estado del desarrollo cultural en las organizaciones a partir del análisis de datos de la realidad y la identificación de proceso sociales básicos (Glaser y Strauss, 1967), empleamos la Grounded Theory o Teoría Fundamentada. Este diseño metodológico fue formulado en 1976 por los sociólogos Barney G. Glaser y Anselm L. Strauss de la Universidad de Columbia y en la actualidad, esta siendo de creciente aplicación en el campo científico de la comunicación y el marketing (Soler Pujals y Fernández Gómez, 2010).

La Teoría Anclada, como también se la conoce, según Strauss "es una metodología de investigación de naturaleza exploratoria con énfasis en la generación y desarrollo de teorías que especifican los fenómenos y las condiciones para manifestarse" (1987: 111). Por tanto estamos ante una metodología que busca la generación de teoría emergente, de carácter inductivo. No se trata de una metodología orientada a la descripción, sino a la conceptualización. Para Goulding (2001) estamos ante un método cualitativo similar a otros como la etnografía (estudio descriptivo e interpretativo 
de la realidad del grupo) ya que las fuentes de información son las mismas: entrevistas, observación de campo y uso de documentos de todo tipo (diarios, cartas, autobiografías, periódicos y otros medios audiovisuales).

En la presente investigación atravesamos distintas fases. La primera de ellas, consistió en definir la muestra teórica, formada por un conjunto de individuos y situaciones claves para el proceso de análisis. La muestra no guarda representatividad probabilística, sino más bien relevancia en cuanto a la amplitud del fenómeno estudiado. El caso de estudio se localizó en España y corresponde a una empresa líder del sector de la distribución (supermercado y Retail) en Europa.

La segunda fase consistió en la recogida de datos. En este sentido, se procedió a confeccionar una base de datos con fuentes múltiples (encuestas, entrevistas, análisis documental, observación). Posteriormente se sucedieron distintas instancias de lectura, discusión y análisis de los mismos para el posterior descubrimiento y etiquetado de conceptos y sus interrelaciones. En el caso de las encuestas, se empleó un dispositivo auto gestionado sobre un muestreo no probabilístico del público interno, como ya mencionamos. El mismo fue distribuido en todos los centros de trabajo durante marzo/abril de 2010. Participaron 2.267 trabajadores, lo que supone una muestra del $40 \%$ del total de colaboradores del grupo empresarial en España. La muestra fue estratificada, compuesta por 73 personas de la gerencia media y alta gerencia, 188 especialistas, 1479 colaboradores y 527 no clasificados. Los centros corresponden a las ciudades españolas de Zaragoza, Burgos, Logroño, Bilbao, Vitoria, Madrid y Barcelona.

El anterior proceso nos permitió formular la idea base, las preguntas a responder y los primeros "memos", a modo de ideas teóricas anotadas que luego consultamos y revisamos durante el transcurso de toda la investigación. Con ello, la siguiente fase fue la de codificación y categorización de los conceptos y sus descriptores. El código es lo más próximo a un concepto teórico, entendiéndolo como la unidad básica de análisis, dado que la teoría se desarrolla a partir de la conceptualización de datos. Las categorías son superiores en nivel y más abstractas que los conceptos que representan. El agrupamiento de conceptos forma categorías. Este proceso, que resulta el más "denso" se basó tanto en los datos en bruto como en la experiencia, incluido el conocimiento de la literatura sobre la cuestión (Corbin y Strauss, 1990).

Posteriormente se procedió a la codificación axial, mediante la cual relacionamos los códigos (categorías y propiedades) 
vía una combinación inductiva y deductiva, reabriendo la búsqueda para validarla o no. Los conceptos seleccionados fueron analizados y reorganizados, con el objetivo de extraer una idea central y sus subordinaciones. En esta fase fue necesario volver al campo, elegir más elementos de análisis o volver al grupo inicial para una nueva búsqueda de datos.

Seguidamente establecimos una codificación selectiva para reducir las categorías iniciales y buscar la variable de núcleo o categoría central. Esta categoría central nos ha permitido establecer el paradigma de la teoría (Goulding, 2001) sobre el objeto de estudio en cuestión, la cultura en las organizaciones.

En conclusión, obtuvimos un conjunto de conceptos con sus propiedades (cualidades y atributos que explican lo que está ocurriendo) relacionados e integrados según las analogías e intereses que comportan. Para este punto tuvimos en cuenta la triangulación que combina distintos métodos o fuentes de datos. A continuación desarrollamos los resultados obtenidos.

\section{Resultados}

\subsection{Matriz de desarrollo cultural organizacional}

El paradigma teórico al que hemos arribado tiene por categoría central la cultura corporativa y denominaremos: matriz de desarrollo cultural organizacional (MADECO). La misma es entendida como la interrelación de los procesos de apropiación de la filosofía organizacional, el sentido de pertenencia, la adaptación, la satisfacción y el liderazgo compartidos por un grupo. Sobre estas categorías se construye el decidir organizacional, que identifica a una organización y la diferencia de otra, haciendo que sus públicos internos se sientan parte constitutiva y constituyente de la misma.

En consecuencia, las categorías y sus correspondientes conceptos de la cultura corporativa que proponemos son:

1. Filosofía.

1.1. Visión.

1.2. Valores.

1.3. Actividades.

2. Pertenencia.

2.1. Lealtad.

2.2. Actitudes.

2.3. Compromiso. 
3. Adaptación.

3.1. Interés.

3.2. Formación.

3.3. Diálogo.

3.4. Puesto de trabajo.

4. Satisfacción.

4.1. Trato.

4.2. Oportunidad.

5. Liderazgo.

5.1. Reconocimiento.

5.2. Conducción.

Figura № 2: Matriz de desarrollo cultural organizacional (MADECO)

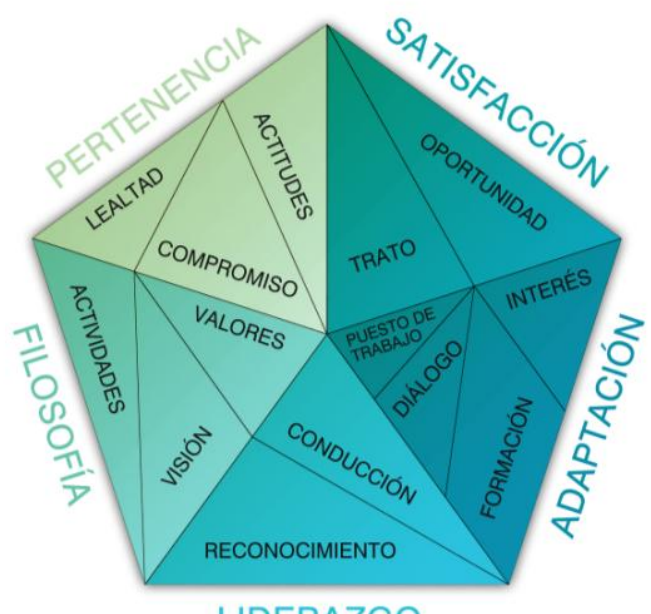

LIDERAZGO

Fuente: elaboración propia.

Cada una de las categorías se construye a partir de conceptos y estos de propiedades o conjunto de atributos e indicadores susceptibles de ser observados y analizados en la realidad organizacional. La matriz MADECO ofrece la posibilidad de diagnosticar el nivel de implantación y desarrollo cultural de una organización a los fines de poder gestionar mediante acciones de comunicación interna su consolidación y crecimiento con vistas a contribuir al proyecto de la organización. Los conceptos no funcionan como compartimientos estancos, sino que por el contrario existe una total interrelación y vínculo constante. La modelización teórica de este conjunto de fenómenos justifica desde el punta de 
vista analítico, y desde luego para su consecuente gestión de mejora constante.

A continuación desarrollamos cada una de las categorías que conforman la MADECO, sus correspondientes variables conceptuales y el conjunto de descriptores que a modo de indicadores permiten observar este fenómeno en la realidad organizacional.

Figura $N^{\circ}$ 3: Niveles de la Matriz de desarrollo cultural organizacional (MADECO)

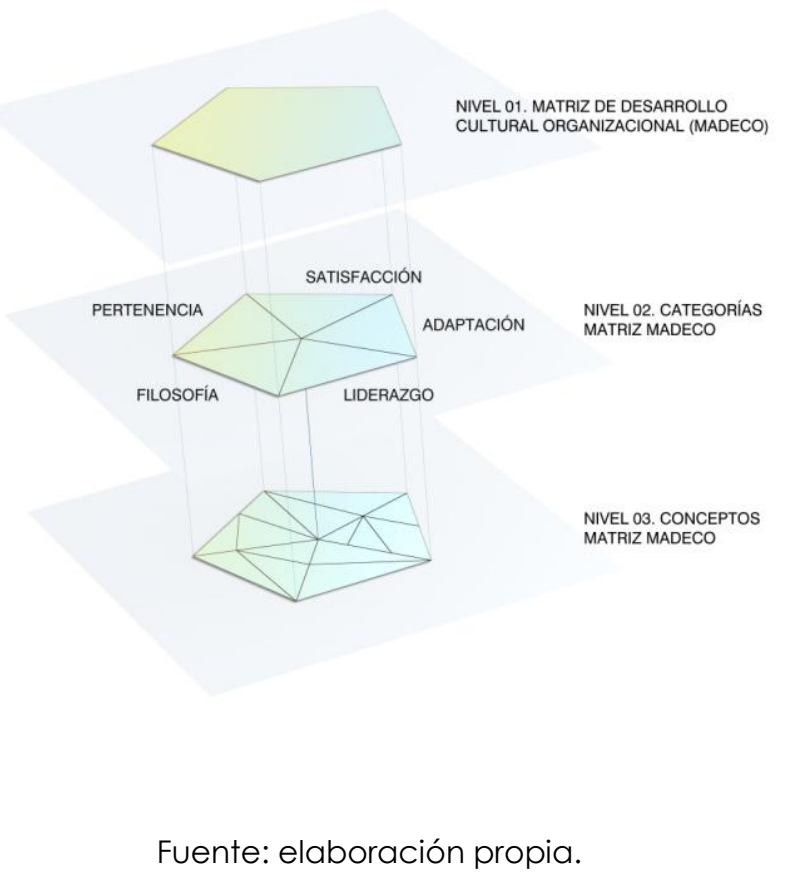

\subsection{Filosofía}

La filosofía se conforma de un conjunto de rasgos y definiciones que constituyen la identidad corporativa. Significa una declaración controlada del ser y querer ser organizacional. Posee tres conceptos: la visión, los valores y las actividades.

La visión guarda relación con el posicionamiento que la organización asume frente: al contexto (mercados, escenarios, etc.); su dinámica (adaptación a los cambios); sus potencialidades; su capacidad innovadora; y posibilidades competitivas.

Algunos de los ítems para medir estos podrían ser: ¿cree que nuestra empresa se está adaptando bien a los cambios que 
ocurren en el mercado?; ila considero como una de las mejores empresas del sector?; ¿̇es una empresa innovadora?; ¿es una empresa líder en el sector de la distribución?

Los valores es el conjunto de convicciones y normas éticas que rigen el comportamiento organizacional. Condicionan axiológicamente la vida de la organización en torno a: su explicita definición y práctica; la confianza que generan en los públicos; el compromiso y la responsabilidad organizacional; la presencia constante de los mismos; y la coherencia discursiva y empírica.

Algunos de los ítems para medir estos podrían ser: ¿es una empresa en la que se puede confiar?; $\dot{\text { se }}$ preocupa por los problemas de la sociedad?; ¿contribuye en acciones de interés social?; ¿̇están presentes en el día a día?; ¿̇es una empresa ética es sus relaciones con el entorno?; ¿̇sus equipos están comprometidos (socialmente, medioambientalmente, en la organización de fiestas populares...) con su entorno?

Finalmente el tercer concepto que define la filosofía organizacional se refiere a las prácticas cotidianas de la organización. Las actividades condicionan los componentes cognitivos y emotivos que priman en una relación, determinando el desempeño actitudinal de cualquier persona. Esta categoría viene a reflejar aquel conjunto de acciones que dan razón de ser y se definen en la misión organizacional. Lo que decimos determina donde imaginamos llegar; lo que hacemos determina lo que somos. Sus propiedades son: el rol que asumimos para con nuestro entorno; las soluciones que aportamos en relación con la satisfacción de necesidades, la generación de valor y aporte de beneficios; las condiciones estructurales y de infraestructura; la calidad y utilidad de los procesos; la calidad y variedad en la oferta de bienes y servicios; el sistema de identidad visual y su correspondiente imagen percibida en los distintos públicos; los modos de hacer y relacionarse; la solidez financiera; la sostenibilidad de la actividad.

Algunos de los descriptores posibles serían: ¿es la empresa más barato de su zona de influencia?; ¿la imagen de la empresa y de los productos es irreprochables?; ¿̇hay un ambiente moderno y cálido?; ¿la compra se hace fácil y rápida?; ¿̇se ofrece un amplio surtido de marca propia?; ¿hay una buena atención al cliente?; ¿̇es una empresa centrada en el cliente?; ¿la calidad de los productos es excelente?; ¿cree que nuestros clientes están muy satisfechos con la oferta y la atención que encuentran en nuestro centro?; ¿qué nuestros clientes reciben un mejor servicio en que en la competencia?; ¿la solidez financiera es muy buena?. 


\subsection{Adaptación}

La adaptación es el primer estadio de implantación cultural, mediante el cual los públicos internos configuran su interés y expectativas respecto a su desempeño y relación con la organización. En esta etapa se adquieren las habilidades y destrezas propias del puesto, rol y funciones asumidas; y articula su lenguaje a la cadena de significaciones, presuposiciones, y competencias comunicativas que definen el discurso social de la propia organización. Posee cuatro conceptos: el interés, la formación, el diálogo y el puesto de trabajo.

El interés viene dado por la necesidad que todo público interno tiene de satisfacer sus necesidades, las cuales motivan su desempeño y relación con la organización. Las propiedades de esta variable vienen dadas en la medida en que la organización muestre interés por el bienestar de sus empleados.

La formación se refiere al conjunto de habilidades y competencias necesarias para cumplir con la función y responsabilidad atribuida. Algunos de los descriptores posibles serían: żtengo los conocimientos y la formación necesaria para hacer bien mi trabajo?; ¿considero que en mi trabajo las múltiples habilidades ayudan a ganar cada día la preferencia de nuestros clientes?; ¿̇la formación que he recibido me ha ayudado mucho a poder realizar mi trabajo cada día mejor?;

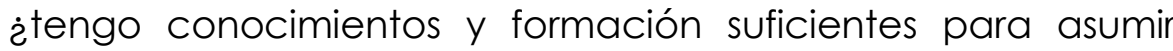
mayores responsabilidades?

En lo que respecta al diálogo, nos referimos a la dirección, el sentido y los soportes que se emplean en los distintos flujos comunicacionales (formales e informales) a través de los cuales circulan los mensajes en la organización. Ya sea los que se suceden en la interrelación de los colaboradores de la propia organización, cómo los que provienen desde el contexto en el cual se desenvuelve.

Algunos de los descriptores posibles serían: ¿̇conozco lo que nuestros clientes opinan de nosotros y lo que debemos hacer

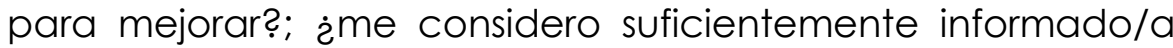
sobre los resultados de la empresa?; $\dot{z}^{m e}$ considero suficientemente informado/a sobre los resultados de mi centro/sección/departamento?; ¿̇conozco los retos y los problemas más importantes que tenemos en mi área/sección/departamento de trabajo?; ¿̇me siento informado/a con los canales de comunicación interna?; ¿comprendo perfectamente los criterios utilizados para la evaluación de mi trabajo? 
Los espacios, su diagramación, la ambientación, la distribución, la infraestructura, los insumos y medios necesarios para la tarea condicionan el desarrollo organizacional y determinan la cultura. Sin embargo, al referimos al concepto de puesto de trabajo, también se corresponde con el rol y la función asignada, las rutinas, las tareas, las responsabilidades, su articulación en la estructura organizativa, el trato y la cordialidad, la retribución percibida, los incentivos y motivaciones, la movilidad, el cumplimiento de objetivos y la percepción de contribución y generación de valor.

Algunos de los descriptores posibles serían: ¿en mi área de trabajo, dedicamos el tiempo suficiente para hablar sobre cómo podemos mejorar la calidad de servicio para los clientes de nuestras tiendas?; ¿̇sé perfectamente qué es lo que la empresa espera de mí, mis responsabilidades están claramente definidas?; ¿̇dispongo de la información que necesito para poder hacer mi trabajo adecuadamente?; ¿dispongo de los medios técnicos adecuados para poder hacer mi trabajo con eficacia?; ¿̇en mi trabajo sé qué hacer para poder influir en el resultado de la prima de progreso?; ¿en mi centro de trabajo realizamos múltiples tareas?; ¿̇en mi centro de trabajo hacemos grupos de progreso para mejorar?; ila cantidad de trabajo que tengo me permite cumplir con mis responsabilidades adecuadamente?

\subsection{Pertenencia}

La pertenencia parte de la consolidación de la adaptación y de un incremento significativo en el nivel de fidelidad, implicación y compromiso para con la organización. El público interno personaliza en sus comportamientos, desempeño y discurso el proyecto institucional, la misión, visión y valores. La percepción de valor debe ser mutua (tanto para la persona como para la organización). En este sentido, el reconocimiento formal, si bien no debe ser condición sine qua non; es muy importante. Este estado de fidelización se puede reconocer en: la lealtad, las actitudes y el compromiso del público interno.

La lealtad supone un contrato moral y tácito que se pone de manifiesto en la relación con la organización y los beneficios (no sólo económicos o materiales) que de ella se obtiene y las perspectivas de continuidad. Algunos de los descriptores posibles serían: ¿̀recomiendo mi empresa como un buen lugar para trabajar?; ¿no busco oportunidades de trabajo en otras empresas?; ¿̇seguiré trabajando aquí al menos durante los dos próximos años?; ¿̇actualmente, si tuviera una oferta de trabajo de otra empresa, no la tendría en cuenta? 
En el caso de las actitudes, el sentido de pertenencia se refuerza ante determinadas conductas colaborativas, proactivas, que no necesariamente estén ceñidas a la función - tareas específicas. El espíritu solidario, optimista, participativo, constructivo son indicadores del desarrollo de actitudes tendientes a consolidar la pertenencia a una organización.

Algunos de los descriptores posibles serían: ¿̇me siento comprometido/a con la resolución de los problemas de los clientes de nuestras tiendas?; ¿̇estoy dispuesto/a a realizar un sobre esfuerzo si mi empresa lo necesita?; ¿d dispongo de autonomía para realizar mi trabajo?; ¿̇cuando en mi área/sección/departamento hay algún problema lo vivo como propio?; ¿̇En la empresa, aunque los resultados sean buenos siempre estamos preocupados por mejorarlos?

El compromiso resulta la más clara manifestación de pertenencia e implicación organización. Además del componente de responsabilidad, el compromiso viene motivado por una clara visualización de oportunidad para la realización y desarrollo personal, profesional. Es una dimensión asociada a un estado pleno de felicidad, ajeno a circunstancialidades y coyunturas. Es sin duda también, una apuesta de futuro que conlleva un conjunto de expectativas y necesidades que deben objetivarse para poder ser gestionadas. Algunos de los descriptores posibles serían: ¿̇me siento responsabilizado/a en mi puesto de trabajo?; ¿̇me siento parte del equipo humano del área en que trabajo?; ¿̇me siento responsable de los resultados que alcanzamos en mi área/sección/departamento de trabajo?; ien mi área/sección/departamento de trabajo, aunque los resultados sean buenos, siempre estamos preocupados por mejorarlos?; ¿̇me siento muy vinculado/a personalmente con las personas del área/sección/departamento en que trabajo?; isiento un gran orgullo de trabajar en mi área/sección/departamento?; ¿̇me siento comprometido/a con la empresa?; ìverdaderamente me siento parte del equipo humano?; ¿̇siento un fuerte vínculo personal hacia la empresa?; ìme siento muy orgulloso/a de trabajar aquí?; ¿̇cuando mi empresa tiene algún problema lo vivo como propio?

\subsection{Liderazgo}

La categoría liderazgo posee dos conceptos, el reconocimiento y la conducción. Esta dimensión de la cultura organizacional se presenta estrechamente vinculada a las aptitudes y actitudes que asuman los cuadros directivos y de conducción. Es una dimensión dinámica, situacional y muy 
compleja de estandarizar, en caso de grandes organizaciones con múltiples emplazamientos y localizaciones, bajo un patrón único. El desempeño personal, la coherencia y perfil de las estructuras de poder condicionan directamente el clima organizacional y en consecuencia el desarrollo cultural de las organizaciones.

La función que tiene el reconocimiento tiene que ver con la motivación para realizar las tareas, el reconocimiento por los logros, progreso, aciertos, al esfuerzo, el aporte, las iniciativas, la participación, el entusiasmo, la confianza. Igualmente, el papel que asume el líder en la gestión de mejoras, los procesos de control de calidad, la gestión por objetivos; también las acciones de evaluación de desempeño, planes de desarrollo y formación deben tener siempre presente el desarrollo de esta variable. Algunos de los descriptores posibles serían: ¿̇me siento muy motivado/a para hacer bien mi trabajo?; ¿mi jefe reconoce el trabajo bien hecho?; ¿confía en sus empleados?

El estudio de la conducción procura identificar el perfil de quienes asumen posiciones de gestión, liderazgo o manejo de grupos. Requiere de la legitimidad de las capacidades para el desempeño del puesto por parte de los conducidos, de los modos de relacionarse, formas y habilidades directivas desplegadas en la práctica, la gestión de objetivos, distribución de funciones, capacidad de trabajo en equipo, visión, delegación, planificación, empatía, etc. Algunos de los descriptores posibles serían: ¿está dirigida por personas muy capaces?; ¿̇considero adecuado el tiempo que me dedica mi jefe para hablar sobre cómo estoy realizando mi trabajo y cómo podemos mejorarlo?; zen mi área/sección/departamento de trabajo nos reunimos periódicamente para hablar de la marcha del trabajo, lo que tenemos que lograr y si lo conseguimos?; ¿̇encuentro a mi jefe cuando lo necesito?; ilas informaciones que me da mi jefe me permiten comprender el porqué de lo que se me pide?; $\dot{z}^{m i}$ jefe respeta mis responsabilidades y deja que yo mismo/a

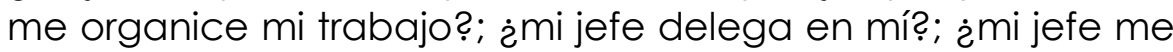
consulta en lo que tiene que ver con mi trabajo?; ¿̇mi jefe dialoga conmigo sobre la valoración que hace de mi trabajo?; ¿en las reuniones individuales con mi jefe para hablar sobre cómo estoy realizando mi trabajo puedo expresar mis ideas?; ¿las reuniones individuales con mi jefe para hablar sobre cómo estoy realizando mi trabajo me resultan útiles?; ¿̇cada vez que hay cambios mi jefe me explica los motivos de los mismos?; ¿̇mi jefe reconoce el derecho al error?; $\dot{m}$ mi jefe promueve y facilita la realización de Grupos de Progreso?; ¿̇considero adecuado el tiempo que me dedica mi jefe para hablar sobre cómo estoy realizando 
mi trabajo y cómo podemos mejorarlo?; ìla retribución variable permite diferenciar de manera muy adecuada la calidad del trabajo de cada persona?

\subsection{Satisfacción}

La categoría satisfacción se subdivide en trato y oportunidad. Se asocia con los niveles de expectativa y superación de objetivos y necesidades por un lado, y con la percepción de un entorno y ambiente de trabajo saludable y sostenible en el tiempo.

En lo que se refiere al trato, corresponde a la satisfacción cotidiana, vinculada a la conciliación con la vida personal, a la calidad y calidez de las relaciones humanas. Algunos de los descriptores posibles serían: ¿̇tiene una reputación excelente en la sociedad?; $\dot{\partial}^{\text {trata }}$ bien a sus empleados?; $\dot{\text { tme siento }}$ satisfecho/a con mi trabajo?; ¿considera que en mi área/sección/departamento trabajamos ahora más eficazmente que en el pasado?; ¿̇creo que la retribución es adecuada en relación con otras empresas del sector?; ¿mi trabajo me permite compaginar razonablemente mi vida personal y profesional, teniendo en cuenta el sector?; $\dot{e}$ es una buena empresa para mí?; ¿̇considero que trabajamos ahora más eficazmente que en el pasado?

Finalmente, la última categoría correspondiente a la satisfacción se refiere a la oportunidad. La misma se sustenta en la coherencia que pueda llegar a existir entre la visión del proyecto personal y el proyecto institucional, y en la confianza que la organización deposita en sus públicos y éstos en la organización. Algunos de los descriptores posibles serían: ¿̇una política responsable de RR.HH. me proporciona oportunidades para mi desarrollo profesional?; ¿̇ofrece oportunidades para la promoción interna?

\section{Conclusiones}

El estudio en profundidad mediante el empleo de la teoría fundamentada nos ha permitido diseñar un modelo teórico de análisis y comprensión de la implantación y el desarrollo de la cultura en las organizaciones, por la cual se configura a partir de la interrelación de los procesos de apropiación de la filosofía organizacional, el sentido de pertenencia, la adaptación, la satisfacción y el liderazgo compartidos por un grupo, sobre los cuales se construye el decidir organizacional, que identifica a una organización y la diferencia de otra, haciendo que sus públicos internos se sientan parte constitutiva y constituyente de ella. La matriz MADECO incluye 
un conjunto de categorías compuestas de conceptos y propiedades $o$ conjunto de atributos $e$ indicadores susceptibles de ser observados y analizados en la realidad organizacional.

Con este aporte, la complejidad que significa el estudio de la cultura encuentra un diseño complejo pero factible de ser empleado en la gestión y mejora organizacional permitiendo individualizar cada uno de los factores psicosociales intervinientes y las relaciones entre los mismos.

\section{Agradecimientos}

Manifestamos nuestro agradecimiento a supermercados Simply (Grupo Auchan) por ofrecer a través de su Cátedra Simply Sano en la Universidad San Jorge, toda su colaboración, no sólo económica, para el desarrollo de esta investigación.

\section{Referencias bibliográficas}

[1] ABRAVANEL, H. ; ALLAIRE, Y.; FIRSIROTU, M. ; HOBBS, B. ; POUPART, R. \& SIMARD, J.J. (1992). Cultura Organizacional, Aspectos teóricos, prácticos y metodológicos. Bogotá: Legis.

[2] ANDRADE RODRÍGUEZ, H. (2003). La cultura Organizacional, administración de recursos simbólicos y comunicación. En La comunicación en las organizaciones. México: Trillas

[3] CORBIN, J. y STRAUSS, A. L. (1990). Basics of qualitative research: Grounded theory procedures and techniques. London, Edit. Sage Publications.

[4] GAGLIARDI, P. (1984). "Creation and change of organizational cultura: A Conceptual Framework". Ponencia presentada en la First Internacional Conference on Organizational Symbolism and Corporate Culture, Lund, Suecia.

[5] GLASER, B. G. (1978). Theoretical sensitivity: Advances in the methodology of grounded theory. Mill Valley, CA, Edit. The Sociology Press.

[6] GLASER, B. G. y STRAUSS, A. L. (1967). The discovery of grounded theory strategies for qualitative research. Chicago, Edit. Aldine Pub.

[7] GONZÁlEZ ROMO, Z. F. (2002). Los ritos y rituales en la cultura corporativa de Wal Mart Supercenter. Tesis de Doctorado. Universitat Autònoma de Barcelona, Facultat de Ciènces de la Comunicació, Departament de Comunicació Audiovisual i de Publicitat, España. 
[8] GOULDING, C. (1999). Consumer research, interpretive paradigms and methodological ambiguities. "European Journal of Marketing", 33(9/10), pp. 859-873.

[9] ---- (2001). Grounded theory: A magical formula or a potential nightmare. "The Marketing Review", 2(1), pp. 21-34.

[10] ---- (2005). Grounded theory, ethnography and phenomenology. "European Journal of Marketing", 39(3/4), pp. 294-308.

[11] SÁNCHEZ QUIRÓS, I. (2010). "El estudio en el campo organizativo. Orígenes antropológicos y congruencia entre el concepto, los niveles de estudio y la metodología de investigación". Cuadernos de Estudios Empresariales 10, 321 341.

[12] SCHEIN, E.H. (1998). La cultura empresarial y el liderazgo. Una visión dinámica. Madrid: Plaza y Janes.

[13] SOLER PUJALS, P. y FERNÁNDEZ GÓMEZ, B. (2010). La Grounded Theory y la investigación cualitativa en comunicación y marketing. Revista Iconol4 [en línea] 1 de Julio de 2010, Año 8, Vol. 2. pp. 203-213. Recuperado (01 de febrero 2010), de

[14] STEFANOVA, E. \& LUCAS, A. (2006). El concepto de cultura en las organizaciones: centralidad actual y evolución histórica. En Brunet, I. \& Alarcón, A. (comps.). Sociología de la empresa y de las organizaciones. (pp. 291-304). Zaragoza: Egido Editorial.

Alejandro ÁLVAREZ-NOBELL

Profesor investigador Universidad San Jorge (USJ) - España aalvarez@usi.es

María ROMERO-CALMACHE

Profesor investigador Universidad San Jorge (USJ) - España mromero@usj.es

Manuel SÁNCHEZ-SANVICENTE Profesor investigador Universidad San Jorge (USJ) - España nsanchez@usj.es

Paz ARAGÜÉS-DUFOL

Profesor investigador Universidad San Jorge (USJ) - España dufoles@gmail.com 
